\title{
3-D hydrodynamic simulations of convection in A stars
}

\author{
O. Kochukhov ${ }^{1}$, B. Freytag ${ }^{1}$, N. Piskunov ${ }^{1}$ and M. Steffen ${ }^{2}$ \\ ${ }^{1}$ Astronomical Observatory, Uppsala University, Box 515, 751 20, Uppsala, Sweden \\ ${ }^{2}$ Astrophysikalisches Institut Potsdam, an der Sternwarte 16, 14482 Potsdam, Germany
}

\begin{abstract}
Broadening and asymmetry of spectral lines in slowly rotating late A-type stars provide evidence for high-amplitude convective motions. The properties of turbulence observed in the A-star atmospheres are not understood theoretically and contradict results of previous numerical simulations of convection. Here we describe an ongoing effort to understand the puzzling convection signatures of A stars with the help of 3-D hydrodynamic simulations. Our approach combines realistic spectrum synthesis and non-grey hydrodynamic models computed with the $\mathrm{CO}^{5} \mathrm{BOLD}$ code. We discuss these theoretical predictions and confront them with highresolution spectra of A stars. Our models have, for the first time, succeeded in reproducing the observed profiles of weak spectral lines without introducing fudge broadening parameters.
\end{abstract}

Keywords. Convection, line: profiles, radiative transfer, stars: atmospheres

\section{Introduction}

A number of complex hydrodynamic processes takes place in the atmospheres of cool A stars $\left(T_{\text {eff }}=7500-8000 \mathrm{~K}\right)$. Convection is arguably the least understood phenomenon, as it presents enormous challenges both for observational detection and theoretical modelling. The atmospheric structure of cool A stars is thought to be close to radiative equilibrium. Nevertheless, prominent signatures of convective motions are observed in several classes of slowly rotating A-type stars. For instance, for Am stars inexplicably broad profiles of strong line are observed. Large microturbulent velocity is often deduced in 1-D model atmosphere analyses and line bisectors show blue asymmetry (Landstreet 1998), which is opposite to the behaviour of the solar-type stars. In cool magnetic Ap stars macroturbulence of $\geqslant 10 \mathrm{~km} \mathrm{~s}^{-1}$ is required to reproduce the width of rare-earth lines formed in the upper atmospheric layers (Kochukhov \& Ryabchikova 2001), suggesting that turbulence is not fully suppressed even in the presence of strong ordered magnetic field. These observations indicate that convection is important for the atmospheric dynamics of A-type stars.

\section{Numerical simulations with $\mathrm{CO}^{5} \mathrm{BOLD}$}

3-D hydrodynamic simulations of convection in A stars are considerably more difficult compared to similar modelling of the solar-type stars. Short radiative time-scales limit the time step of simulation and thus require 10 to 100 more computing time for an A-type star compared to the corresponding simulation of the solar-type granulation. Furthermore, simulations need to resolve the steep temperature jump below the photosphere and have to cover simultaneously the small surface granules and the large deep cells.

Here we develop further the 3-D A-star model of Freytag \& Steffen (2004) and present first results of the new study that has finally overcome computational problems associated with simulating the A-star convection zone. 

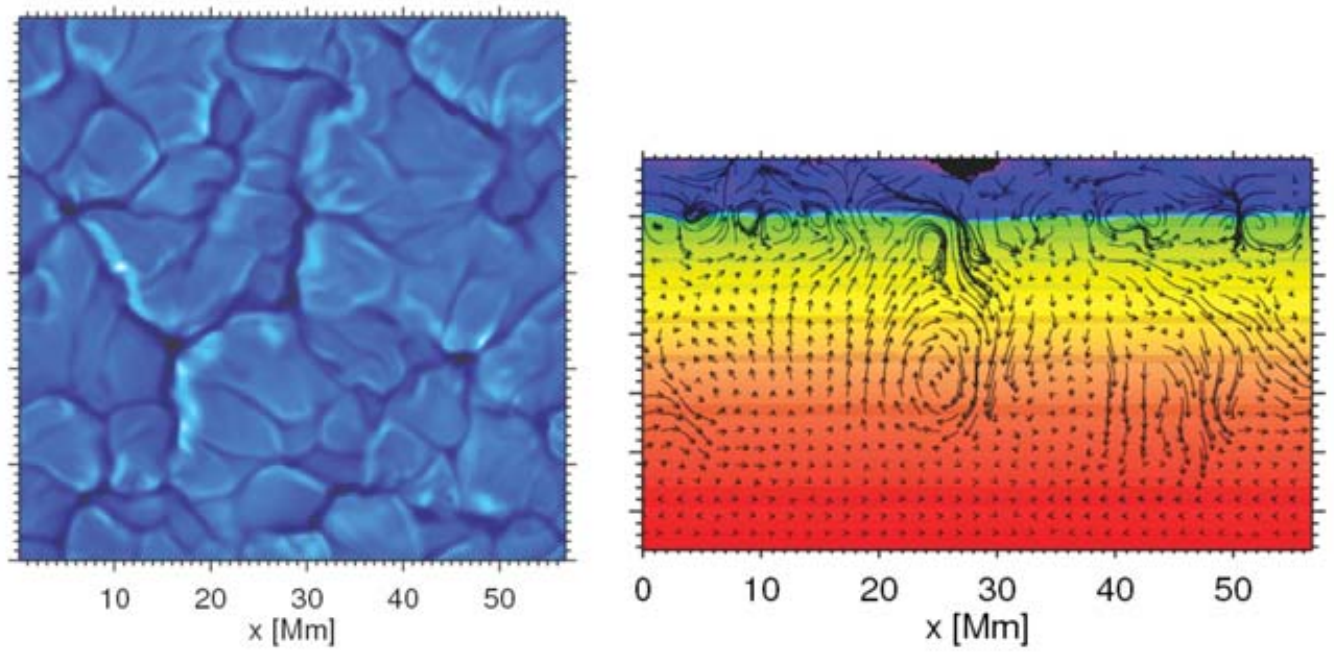

Figure 1. Left panel: continuum intensity resulting from the 3-D hydrodynamic simulations of A-star convection. Right panel: illustration of the vertical structure of the 3-D model. Arrows represent velocity field, greyscale plot shows temperature distribution.

We have used the $\mathrm{CO}^{5}$ BOLD code (Freytag 2004, Wedemeyer et al. 2004) to perform 3-D simulations of the surface convection zone in a main sequence A-type star $\left(T_{\text {eff }}=8000 \mathrm{~K}, \log g=4.0\right)$. For solving hydrodynamics equations $\mathrm{CO}^{5} \mathrm{BOLD}$ employs conservative finite volume approach. 3-D radiation transport is based on a modified Feautrier scheme using long characteristics. We use grey or wavelength dependent opacities derived from ATLAS6 opacity distribution functions. Simulations are carried out in Cartesian geometry, assuming periodic lateral boundary conditions. Top and bottom boundaries are closed for flows and transparent for radiation. $220 \times 220 \times 170$ grid points are used, corresponding to the simulation box with size $57 \times 57 \times 34.5 \mathrm{Mm}$.

Fig. 1 illustrates the typical surface granulation pattern and the vertical structure of the velocity field deduced with $\mathrm{CO}^{5} \mathrm{BOLD}$.

\section{Spectrum synthesis and comparison with observations}

A new 3-D spectrum synthesis code (Piskunov, in preparation) was used to compute line profiles from the $\mathrm{CO}^{5} \mathrm{BOLD}$ snapshots. For A-star models we used the LTE source function and computed line and continuum opacities as in 1-D Synth code by Piskunov (1992). Radiative transfer is solved with a short characteristic formal solver based on the quadratic Bezier-spline approximation of the source function. The flux spectra are produced from the intensity profiles computed for 29 directions.

We compared our theoretical spectra with the high-resolution observations of the slowly rotating A2m star HR $4750\left(T_{\text {eff }}=8100 \mathrm{~K}, \log g=4.1\right.$, Landstreet 1998). A set of Fe and $\mathrm{Cr}$ spectral lines in the $4612-4645 \AA$ wavelength region was synthesized, adopting chemical abundances, stellar and atomic parameters from Landstreet (1998).

Fig. 2 presents a comparison of observations and 3-D spectrum synthesis for selected spectral lines. Our 3-D model reproduces the line cores of strong spectral features, but fails to fit the observed wide wings and strong blueward asymmetry. On the other hand, weak lines are successfully reproduced. In contrast to the solar-type stars, bisectors computed for our 3-D hydrodynamic models of A stars are not skewed to the red. In our 


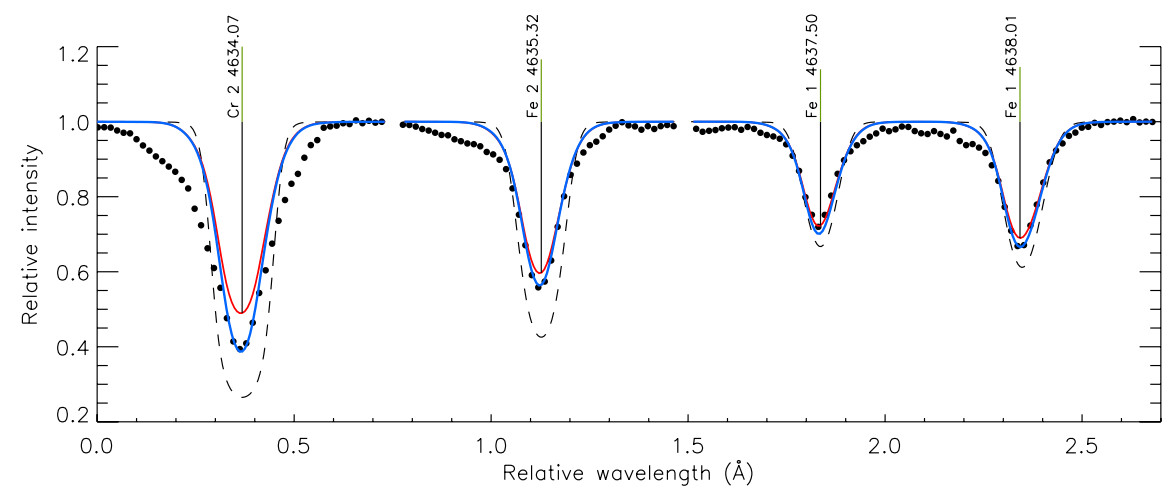

Figure 2. Comparison of the observed spectrum of Am star HR 4750 (symbols) with the theoretical line profiles computed using grey (thin solid curve) and non-grey (thick solid curve) $\mathrm{CO}^{5} \mathrm{BOLD}$ models. The dashed line shows $1-\mathrm{D}$ spectrum synthesis using microturbulence $\xi_{t}=3 \mathrm{~km} \mathrm{~s}^{-1}$ and no macroturbulent or rotational broadening.

calculations, the resulting bisectors span a small velocity range and have a weak blue asymmetry in the outer wings - a trend which agrees with observations.

\section{Conclusions}

We have carried out 3-D numerical simulations of the A-star convection zone using the radiation hydrodynamics code $\mathrm{CO}^{5} \mathrm{BOLD}$. These are the first $3-\mathrm{D}$ RHD calculations for A stars which employ non-grey opacities. The granulation pattern predicted for A stars appears to be generally similar to the one found in the solar-type stars, albeit with some distinctive features (bright rims of granules, large horizontal velocity component). Comparison of the detailed 3-D spectrum synthesis with $\mathrm{CO}^{5}$ BOLD snapshots and highresolution observations shows that our 3-D model successfully reproduces the shape of weak lines in the Am star HR 4750. This is the first time when a 3-D RHD model is able to fit the A-star line profiles without introducing fudge line broadening parameters. Theoretical models fail to reproduce the broad wings and prominent blueward asymmetry observed in the strong spectral lines, indicating that further improvements (realistic abundances, better resolution) of 3-D models are required.

\section{Acknowledgements}

OK thanks IAU and the Czech Academy of Sciences for supporting his participation in the 26th IAU General Assembly. This work was also supported by the travel grant from the Royal Swedish Academy of Sciences. We thank Prof. J. Landstreet for providing us with the high-resolution observations of A stars.

\section{References}

Freytag, B., Steffen, M., Wedemeyer, S., \& Ludwig, H.-G. 2004, CO $50 L D$ User Manual, http://www.astro.uu.se/ $\mathrm{bf} /$ co5bold_main.html

Freytag, B. \& Steffen, M. 2004, in The A-Star Puzzle, eds. J. Zverko, J. Žižňovský, S.J. Adelman, W.W. Weiss, IUAS 224, 139

Kochukhov, O. \& Ryabchikova, T. 2001, A\&A 374, 615

Landstreet, J.D. 1998, A\& $A$ 338, 1041

Piskunov, N. 1992, in Stellar Magnetism, eds. Yu.V. Glagolevskij, I.I. Romanyuk, St. Petersburg, 92

Wedemeyer, S., Freytag, B., Steffen, M., Ludwig, H.-G., \& Holweger, H. 2004, A\&A 414, 1121 